\title{
Reducing Quantity Hallucinations in Abstractive Summarization
}

\author{
Zheng Zhao Shay B. Cohen Bonnie Webber \\ Institute for Language, Cognition and Computation \\ School of Informatics, University of Edinburgh \\ 10 Crichton Street, Edinburgh, EH8 9AB \\ zheng.zhaoded.ac.uk, \{scohen, bonnie\}@inf.ed.ac.uk
}

\begin{abstract}
It is well-known that abstractive summaries are subject to hallucination-including material that is not supported by the original text. While summaries can be made hallucinationfree by limiting them to general phrases, such summaries would fail to be very informative. Alternatively, one can try to avoid hallucinations by verifying that any specific entities in the summary appear in the original text in a similar context. This is the approach taken by our system, HERMAN. The system learns to recognize and verify quantity entities (dates, numbers, sums of money, etc.) in a beamworth of abstractive summaries produced by state-of-the-art models, in order to up-rank those summaries whose quantity terms are supported by the original text. Experimental results demonstrate that the ROUGE scores of such up-ranked summaries have a higher Precision than summaries that have not been upranked, without a comparable loss in Recall, resulting in higher $F_{1}$. Preliminary human evaluation of up-ranked vs. original summaries shows people's preference for the former.
\end{abstract}

\section{Introduction}

Automatic summarization is the task of compressing a lengthy text to a more concise version that preserves the information of the original text. Common approaches are either extractive, selecting and assembling salient words, phrases and sentences from the source text to form the summary (Lin and Bilmes, 2011; Nallapati et al., 2017; Narayan et al., $2018 \mathrm{~b}$ ), or abstractive, generating the summary from scratch, containing novel words and phrases that are paraphrased from important parts of the original text (Clarke and Lapata, 2008; Rush et al., 2015; Wang et al., 2019). The latter is more challenging as it involves human-like capabilities, e.g., paraphrasing, generalizing, inferring and including

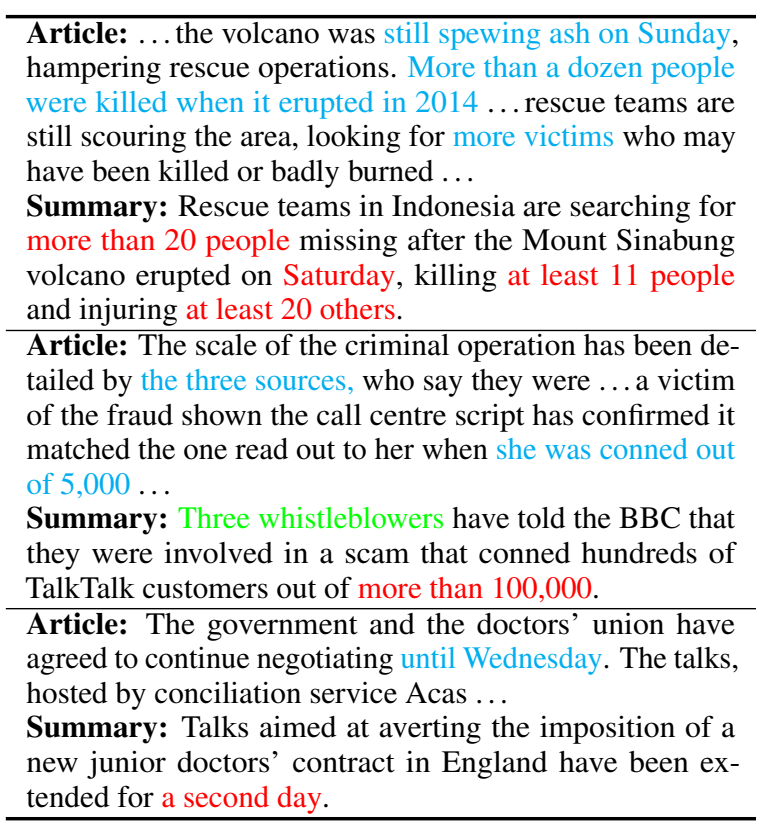

Table 1: Examples of system generated abstractive summaries with hallucinated quantities. Phrases in the articles highlighted in cyan have been used by the summarization system to generate summaries. Phrases in the summaries highlighted in green are correct with respect to the article, whereas red highlighting indicates hallucinations. Note that the first article describes both a new eruption and a previous one in 2014. It was in the previous eruption that more than a dozen people were killed, hence a hallucination of at least 11 people killed and at least 20 injured in the new eruption.

real-world knowledge (See et al., 2017).

Abstractive summarization has attracted increasing attention recently, thanks to the availability of large-scale datasets (Sandhaus, 2008; Hermann et al., 2015; Grusky et al., 2018; Narayan et al., 2018a) and advances on neural architectures (Sutskever et al., 2014; Bahdanau et al., 2015a; Vinyals et al., 2015; Vaswani et al., 2017). Although modern abstractive summarization systems generate relatively fluent summaries, recent work has called attention to the problem they have with 
factual inconsistency (Kryscinski et al., 2019a). That is, they produce summaries that contain hallucinated facts that are not supported by the source text. A recent study has shown that up to $30 \%$ of summaries generated by abstractive summarization systems contain hallucinated facts (Cao et al., 2018). Such high levels of factual hallucination raise serious concern about the usefulness of abstractive summarization, especially if one believes that summaries (whether extractive or abstractive) should contain a mixture of general and specific information (Louis and Nenkova, 2011).

This paper explores reducing the frequency of one type of hallucinated fact in abstractive summaries-hallucinated quantities. We focus on quantities not only because they are important for factual consistency, but also because, unless they are wildly inaccurate, a reader might not notice that they are hallucinated. Moreover, unlike people's names (which are also frequently hallucinated), quantity entities are rarely referred to anaphorically, avoiding the need to resolve anaphoric expressions, making them an excellent testbed for the study of hallucination. The quantities we address can be broadly categorized into seven types: dates, times, percentages, monetary values, measurements, ordinals, and cardinal numbers. Table 1 shows some examples of hallucinated quantities introduced by abstractive summarization models.

We present HERMAN ${ }^{1}$, a system that learns to recognize quantities in a summary and verify their factual consistency with the source text. Our system can be easily coupled with any abstractive summarization models that produce a beam-worth of candidate summaries. After verifying consistency, we use a re-ranking approach that up-rank those summaries whose quantities are supported by the source text, similar to the method proposed by Falke et al. (2019). Training data is automatically generated in a weakly supervised manner from a summarization dataset containing both original and synthetic data. The synthetic data is created by selecting quantity entities from the summary and replacing them with randomly selected entities from the source text that are the same type. We perform experiments on the XSum dataset (Narayan et al., 2018a) which favors an abstractive modeling approach. Results based on automatic evaluation using ROUGE (Lin, 2004) demonstrate that

\footnotetext{
${ }^{1}$ Name inspired by the fact-checker Herman Brooks from the 1980s American sitcom "Herman's Head."
}

up-ranked summaries have higher ROUGE Precision than original summaries produced by three different summarization systems. While ROUGE Recall of these up-ranked summaries is lower, overall ROUGE $F_{1}$ is higher for up-ranked summaries, showing that it is not simply a like-for-like tradeoff of Recall for Precision. A preliminary human evaluation study shows that subjects prefer the upranked summaries to the original summaries.

\section{Related Work}

Recent studies have suggested that abstractive summarization systems are prone to generate summaries with hallucinated facts that cannot be supported by the source document. Cao et al. (2018) reported that almost $30 \%$ of the outputs of a state-ofthe-art system contain factual inconsistencies. An evaluation of summaries produced by recent stateof-the-art models via crowdsourcing suggested that $25 \%$ of the summaries have factual errors (Falke et al., 2019). The work also showed that ROUGE scores do not correlate with factual correctness, emphasizing that ROUGE based evaluation alone is not enough for summarization task. In addition, Kryscinski et al. (2019a) pointed out that current evaluation protocols correlate weakly with human judgements and do not take factual correctness into account. Maynez et al. (2020) conducted a large scale human evaluation on the generated summaries of various abstractive summarization systems and found substantial amounts of hallucinated content in those summaries. They also concluded that summarization models initialized with pre-trained parameters perform best on not only ROUGE, but also human judgements of faithfulness/factuality.

Another line of research focused on evaluating factual consistency of summarization systems. Kryscinski et al. (2019b) proposed a weaklysupervised, model-based approach for evaluating factual consistency between source documents and generated summaries. They first generate training data by applying a series of transformations to randomly selected individual sentences from source documents (which they call claims) and assign them a binary label based on the type of the transformation. Then they train a fact-checking model to classify the label of the claim and extract spans in both the source document and the generated summary explaining the model's decision. Goodrich et al. (2019) introduced a model-based 


\begin{tabular}{|c|c|c|c|c|c|c|c|c|c|c|}
\hline Article & $\begin{array}{l}\text { The cras } \\
\text { ambular } \\
\text { the exte } \\
\text { are ong }\end{array}$ & $\begin{array}{l}\text { h happen } \\
\text { ce was se } \\
t \text { of their } \\
\text { ing into } t\end{array}$ & $\begin{array}{l}\mathrm{d} \text { at } \mathrm{Ev} \\
\text { th to the } \\
\text { injuries } \\
\text { is matt }\end{array}$ & $\begin{array}{l}\text { anton a } \\
\text { scene } \\
\text { was n }\end{array}$ & $\begin{array}{l}\text { tabout } 1 \\
\text { The occ } \\
\text { t known, } \\
\text { to furthe }\end{array}$ & $\begin{array}{l}\text { :20 c } \\
\text { upant } \\
\text { polic }\end{array}$ & $\mathrm{nSa}$ & $\begin{array}{l}\text { turday. Th } \\
\text { all three ve } \\
\text { d. A spoke } \\
\text { are sough }\end{array}$ & $\begin{array}{l}\text { fire service } \\
\text { icles were } \\
\text { man added }\end{array}$ & $\begin{array}{l}\text { and the air } \\
\text { injured, but } \\
\text { : "Inquiries } \\
\text { "... }\end{array}$ \\
\hline Summary & Several & people & have & been & injured & in & $\mathrm{a}$ & three-car & collision & on \\
\hline$Y$ labels & $B-V$ & 0 & O & 0 & O & 0 & 0 & $B-V$ & 0 & 0 \\
\hline$M$ labels & 1 & 0 & 0 & 0 & 0 & 0 & 0 & 1 & 0 & . \\
\hline$z$ label & \multicolumn{10}{|c|}{ VERIFIED } \\
\hline
\end{tabular}

Table 2: An example of a VERIF IED summary with its labels from our dataset. Cyan text highlights the support in the source document for the quantity token highlighted green in the summary.

metric for estimating the factual accuracy of generated text. Factual accuracy is defined as Precision between claims made in the source document and the generated summary, where claims are represented as subject-relation-object triplets. They also released a new dataset for training relation classifiers and end-to-end fact extraction models based on Wikipedia and Wikidata.

Several studies have focused on tackling the problem of factual inconsistencies between inputs and outputs of summarization models by exploring different model architectures and methods for training and inference. Cao et al. (2018) attempted to solve the problem by encoding extracted facts as additional inputs to the system. The fact descriptions are obtained by leveraging Open Information Extraction (Banko et al., 2007) along with parsed dependency trees of the input text. Zhang et al. (2019) developed a framework to evaluate the factual correctness of generated summaries by employing an information extraction module to check facts against the source document, and proposed a training strategy that optimizes the model using reinforcement learning with factual correctness as a reward policy. Falke et al. (2019) proposed a re-ranking approach to improve factual consistency of summarization models. Their approach used natural language inference (NLI; Bowman et al. 2015) models to score candidate summaries obtained in beam search by averaging the entailment probability between all sentence pairs of source document and summary. The summary with the highest score is up-ranked and used as final output of the summarization system. After evaluating their approach using summaries generated by summarization systems trained on the CNN-DailyMail corpus (Hermann et al., 2015), they concluded that out-of-the-box NLI models transfer poorly to the task of evaluating factual correctness, limiting the effectiveness of re-ranking.

\section{Methodology}

Let $X$ be the article and $S$ be the corresponding summary where both are sequences of tokens, $x_{1} \cdots x_{a}$ and $s_{1} \cdots s_{n}$, respectively. Given a $(X, S)$ pair, our aim is to generate a tag sequence $Y$ with the same length as $S$ (i.e., $n$ ) and a summarylevel label $z \in\{$ VERIFIED, UNVERIFIED $\}$, indicating whether the summary $S$ can be verified using $X$. The generated tag sequence $y_{1} \cdots y_{n}$ contains token-level labels where $y_{j} \in\{\mathrm{B}-\mathrm{V}, \mathrm{B}-\mathrm{U}$, $I-U, I-V, O\}$ indicating whether the token is Verified, Unverified, or Other. We adopt the BIO format (Ramshaw and Marcus, 1999) for labels since entities may span multiple tokens. To aid the recognition of quantity based entities, we also obtain a sequence of binary labels $M=\left(m_{1}, \ldots, m_{n}\right)$ for the summary indicating the location of these entities.

Our approach consists of two steps. First, we create a synthetic, weakly-supervised dataset $\mathcal{D}=$ $\left\{\left(X^{(i)}, S^{(i)}, M^{(i)}, Y^{(i)}, z^{(i)}\right) \mid i \in\{1 \ldots N\}\right\}$ consisting of $N$ input-output pairs, where $X, S$, and $M$ are the input, $Y$ and $z$ are the output. At training time, a verification model learns to recognize and verify quantities in the summary. At test time, the same verification model is applied to the summaries identified in a beam search for candidate summaries carried out by the summarization systems, which results in each of them being given a verification score. We provide a detailed description in the rest of this section.

\subsection{Dataset Generation}

The dataset used to train the verification model comprises the dataset used to train the summarization system, augmented with negative examples and additional labels. As we focus on quantities, 


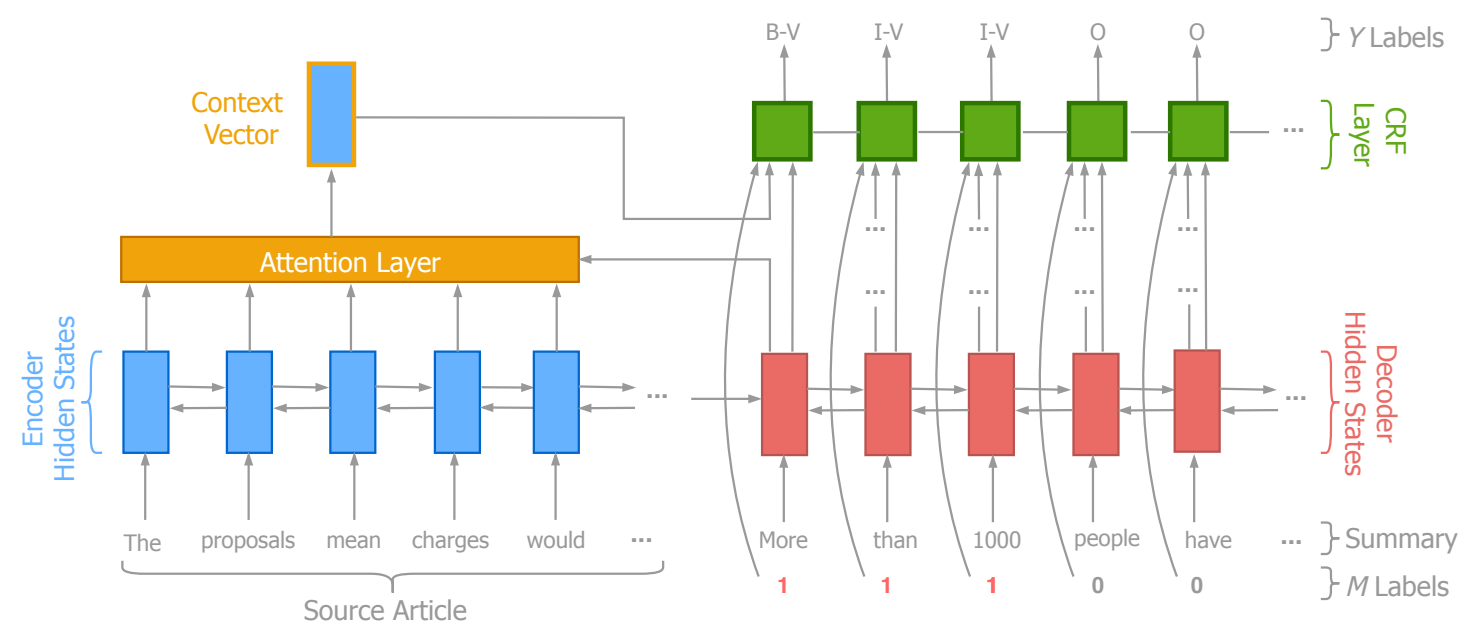

Figure 1: Architecture of HERMAN. Note that the binary classifier for predicting whether a summary is verified $(z$ labels) is omitted here. It simply takes the context vectors of the summary tokens and run through a MLP classifier.

we apply the spaCy NER tagger (Honnibal and Montani, 2017) to identify all such entities in both the article and summary. A gold summary in the original summarization dataset receives a $z$ label VERIF IED. To generate versions of this summary with $z$ label UNVERIFIED, we replace quantity entities in the summary with randomly selected entities from the article that are the same type. For example, a date entity can only be replaced by another date entity from the article. We ensure the UNVERIFIED summary is different from its VERIFIED counterpart. If an article only contains the one quantity entity which appears in the VERIF IED summary, i.e. no replacement can be found to get the UNVERIFIED version, we discard both examples for our dataset to maintain a balanced dataset.

In addition to the binary summary-level label $z$, we also generate two sequences of labels $Y$ and $M$. Quantity entities recognized by spaCy NER in VERIF IED summaries are labeled $V$, and replaced ones in the UNVERIFIED summaries are labelled $\mathrm{U}$. Tokens with $\bigcirc$ labels are unlikely to directly affect whether a quantity based entity has been hallucinated, whereas tokens with $\mathrm{V}$ and $\mathrm{U}$ labels indicate they are important and could potentially affect the factual accuracy of the summary. With BIO format adopted, these labels become $B-V, B-U, I-V$, $\mathrm{I}-\mathrm{U}$, and $\mathrm{O}$. For the sequence of binary labels $M$ :

$$
m_{j}=\left\{\begin{array}{ll}
0, & \text { if } y_{j}=0 \\
1, & \text { otherwise }
\end{array} .\right.
$$

Table 2 illustrates an example of VERIF IED sum- mary with its labels and corresponding article.

\subsection{Verification Model}

The overall architecture for our verification model HERMAN is illustrated in Figure 1. The article encoder provides hidden representations for every input token which are then fed to a decoder with attention to obtain the context vector. The context vectors from every token in the summary are then fed into a Conditional Random Fields (CRF) layer (Lafferty et al., 2001) to generate the tag sequence $Y$. The same context vectors are fed into a binary classifier to obtain the binary label $z$.

BiLSTM Article Encoder For input article $X$ where $X=\left\{x_{1}, \ldots, x_{a}\right\}$ and $x_{i}$ denotes the $i$ th token in $X$, a contextualized token-level encoding $h_{i}$ is obtained via a BiLSTM encoder (Hochreiter and Schmidhuber, 1997):

$$
\begin{gathered}
\vec{h}_{i}=\operatorname{LSTM}_{f}\left(x_{i}, \vec{h}_{i-1}\right), \\
\overleftarrow{h}_{i}=\operatorname{LSTM}_{b}\left(x_{i}, \overleftarrow{h}_{i+1}\right) \\
h_{i}=\left[\vec{h}_{i} ; \overleftarrow{h}_{i}\right]
\end{gathered}
$$

where $\vec{h}_{i}$ and $\overleftarrow{h}_{i}$ are hidden states of forward and backward LSTMs at time step $i$, and ; denotes the concatenation operation.

BiLSTM-CRF Decoder with Attention The decoder generates sequence of labels $Y$ as well as a binary label $z$. As the length of labels to be decoded is fixed, the setup is similar to BiLSTM-CRF used in the sequence tagging task (Huang et al., 2015). The difference is that the decoder takes additional 
input $h_{i}$ which is article encoding and incorporates attention mechanism (Bahdanau et al., 2015b). The BiLSTM with attention component first encodes the summary, token by token, to produce an intermediate representation. We also obtain a sequence of binary labels $M=\left\{m_{1}, \ldots, m_{n}\right\}$ for the summary using spaCy NER to recognize tokens that make up quantity entities. Then the intermediate representation, along with the binary label sequence, is fed to the CRF layer to predict the $Y$ label. The intermediate representation is also fed to an MLP classifier to obtain the binary label $z$.

\subsection{Training and Inference}

Given the training set with labelled sequence $\left\{X^{(i)}, S^{(i)}, M^{(i)}, Y^{(i)}, z^{(i)} \mid i \in\{1 \ldots N\}\right\}$, we maximize the conditional log likelihood for the local verification objective:

$\bar{w}=\underset{w}{\operatorname{argmax}} \sum_{i=1}^{N} \log p\left(Y^{(i)} \mid X^{(i)}, S^{(i)}, M^{(i)}, w\right)$,

where $w$ denotes the model's parameters including the weights of the LSTMs and the transition weights of the CRF. The loss function for $Y$ labels is the negative log-likelihood based on $Y^{(i)}=\left\{y_{1}, \ldots, y_{n}\right\}$ :

$$
\mathcal{L}_{Y}=-\sum_{i=1}^{N} \sum_{j=1}^{n} \log p\left(y_{j}\right),
$$

where $y_{j} \in Y^{(i)}$. For global verification which is predicting $z$ label, the loss function is the binary cross entropy:

$$
\begin{aligned}
\mathcal{L}_{z}=\sum_{i=1}^{N} z^{(i)} & \log p\left(z^{(i)}\right) \\
& +\left(1-z^{(i)}\right) \log \left(1-p\left(z^{(i)}\right)\right) .
\end{aligned}
$$

The final objective which combines both local and global verification is defined as the following:

$$
\mathcal{L}=\alpha \mathcal{L}_{Y}+(1-\alpha) \mathcal{L}_{z},
$$

where $\alpha \in[0,1]$ is a hyperparameter indicating weight balance between $\mathcal{L}_{Y}$ and $\mathcal{L}_{z}$. At test time, inference for a summary $S$ is obtained by applying Viterbi algorithm at the CRF layer to find the most probable sequence $\hat{Y}$ :

$$
\hat{Y}=\underset{Y}{\operatorname{argmax}} P(Y \mid X, S, M, \bar{w}) .
$$

\subsection{Re-ranking to Avoid Hallucination}

We adopt a re-ranking approach in order to reduce the frequency of hallucinated quantities in the output of abstractive summarization. This is similar to the approach taken by Falke et al. (2019) with the difference being that their system's inputs are sentence level whereas ours are document-level. Assume an abstractive summarization system can produce a list of $k$ candidate summaries $S_{1}, \ldots, S_{k}$ for a given document $X$ using beam search, we leverage predictions of HERMAN to give each summary a verification score. Our scoring approach has two variants: HERMAN-GLOBAL, and HERMANLOCAL. HERMAN-GLOBAL uses the raw output of global verification label $z$ which has a real value between $[0,1]$. HERMAN-LOCAL uses the average probabilities of $\mathrm{B}-\mathrm{V}, \mathrm{B}-\mathrm{U}, \mathrm{I}-\mathrm{V}$, and $\mathrm{I}-\mathrm{U}$ labels where entries of $B-U$ and $I-U$ are counted negatively. Out of the $k$ candidate summaries, the summary with the highest verification score is selected as the final generated summary for the summarization system.

\section{Dataset}

We use the XSum dataset which was developed for abstractive document summarization (Narayan et al., 2018a). The XSum dataset consists of BBC articles, with a single-sentence summary of each. This summary is a professionally written introductory sentence, typically written by the author of the article, which is separated from the article, with the remaining text taken to be the document. This one-sentence summary, different from a headline whose purpose is to attract readers to read the article, draws on information distributed in various parts of the document and displays multiple levels of abstraction including paraphrasing, fusion, synthesis, and inference. The dataset contains 204,045 instances for training, 11,332 instances for validation, and 11,334 instances for testing. Overall, 55\% of the instances contain at least one quantity. The distribution of quantity entities is shown in Table 3 . It is clear that the different types of quantities are distributed unevenly: While almost $30 \%$ of summaries contain at least one date entity, only $1 \%$ contain at least one quantity entity. Due to the way in which the summary was created for a document, the summary often contains phrases that do not appear in the document itself. In fact, fewer than $16 \%$ of the summaries in the test set have quantity tokens that also appear in their corresponding 


\begin{tabular}{ccccccc}
\hline Date & Time & Percent & Money & Quantity & Ordinal & Cardinal \\
$29 \%$ & $2 \%$ & $1 \%$ & $4 \%$ & $1 \%$ & $8 \%$ & $25 \%$ \\
\hline
\end{tabular}

Table 3: The distribution of quantity entities in the XSum dataset. Note that the percentages sum to more than $55 \%$, as a summary can contain more than one type of quantity entity. For more details regarding the types of entities, please refer to the official spaCy webpage ${ }^{2}$.

documents.

In order to obtain the dataset used to train HERMAN, we follow procedures described in Section 3.1. We apply same pre-processing steps noted by Narayan et al. (2018a). We also truncate the input document to 400 tokens and limit the length of the summary to 90 tokens. The dataset size for training, validation, and test are 190,370, 10,594, and 10,592, respectively. As noted in Section 3.1, the dataset we use is smaller than the XSum dataset because we discard instances which cannot be perturbed to obtain an UNVERIF IED summary.

\section{Experiments}

For all experiments, we set the hidden dimensions to 256 , the word embeddings to 100 , and the vocabulary size to $50 \mathrm{k}$. The word embeddings are initialized using pre-trained GloVe (Pennington et al., 2014) vectors (6B tokens, uncased). We also experimented using a pre-trained, base-uncased BERT (Devlin et al., 2019) for word embedding initialization. Our training used the Adam optimizer (Kingma and $\mathrm{Ba}, 2015$ ) with a learning rate of 0.001 . We also use gradient clipping with a maximum gradient norm of 5 and we do not use any kind of regularization. We use loss on the validation set to perform early stopping. We set $\alpha$ to 0.66 , suggesting local verification is more important than global verification. Our model was trained on a single GeForce GTX 1080 Ti GPU with a batch size of 32. We use PyTorch (Paszke et al., 2019) for our model implementation. For CRF, we used the AllenNLP library (Gardner et al., 2018) with constrained decoding for the BIO scheme. To evaluate our verification model, we need outputs from abstractive summarization systems. We obtain those from three selected systems: TCONVS2S (Narayan et al., 2018a), BERTSUM (Liu and Lapata, 2019), and BART (Lewis et al., 2019) using pre-trained checkpoints provided by the authors.

\footnotetext{
${ }^{2}$ https://spacy.io/api/annotation\# named-entities
}

\begin{tabular}{cccc}
\hline Label & Precision & Recall & $\mathrm{F}_{1}$ \\
\hline $\mathrm{B}-\mathrm{V}$ & 75.18 & 78.13 & 76.63 \\
$\mathrm{~B}-\mathrm{U}$ & 75.11 & 71.28 & 73.14 \\
$\mathrm{I}-\mathrm{V}$ & 84.78 & 85.63 & 85.20 \\
$\mathrm{I}-\mathrm{U}$ & 83.86 & 83.93 & 83.89 \\
$\mathrm{O}$ & 100.0 & 100.0 & 100.0 \\
\hline
\end{tabular}

Table 4: Results of HERMAN on the test set using GloVe word embedding.

\begin{tabular}{cccc}
\hline Label & Precision & Recall & $\mathrm{F}_{1}$ \\
\hline $\mathrm{B}-\mathrm{V}$ & 72.83 & 81.24 & 76.81 \\
$\mathrm{~B}-\mathrm{U}$ & 75.73 & 69.28 & 72.37 \\
$\mathrm{I}-\mathrm{V}$ & 84.58 & 87.27 & 85.90 \\
$\mathrm{I}-\mathrm{U}$ & 85.03 & 83.47 & 84.24 \\
$\mathrm{O}$ & 100.0 & 100.0 & 100.0 \\
\hline
\end{tabular}

Table 5: Results of HERMAN on the test set using BERT word embedding.

\section{Results}

Automatic Evaluation We first present results in Table 4 from our verification model using GloVe on the test set. On the binary classification task of determining whether a summary is VERIFIED or UNVERIFIED, the model achieved accuracy of 80.12 and $F_{1}$ of 80.94. The results using BERT are displayed in Table 5. The model attained accuracy of 80.23 and $F_{1}$ of 81.6. While no significant difference can be observed in performance, using BERT does triple the needed training time, so does not seem justified.

The standard automatic evaluation metric for summarization is ROUGE. We report the Precision, Recall and $\mathrm{F}_{1}$ scores of ROUGE-1/2/L, which respectively measure the word-overlap, bigramoverlap, and longest common sequence between system and reference summaries. Using HERMAN, we obtain verification scores for the full beam of candidate summaries produced by the summarization systems. We re-rank candidate summaries using the verification score as described in Section 3.4 and evaluate the up-ranked summaries. In addition to HERMAN-GLOBAL and HERMAN- 


\begin{tabular}{|c|c|c|c|c|c|c|c|c|c|c|c|}
\hline & Model & $\mathrm{R} 1-\mathrm{R}$ & R1-P & $\mathrm{R} 1-\mathrm{F}$ & $\overline{\mathrm{R} 2-\mathrm{R}}$ & $\overline{\mathrm{R} 2-\mathrm{P}}$ & $\overline{\mathrm{R} 2-\mathrm{F}}$ & RL-R & RL-P & $\overline{\mathrm{RL}-\mathrm{F}}$ & avg-Q \\
\hline \multirow{5}{*}{$\begin{array}{l}\stackrel{E}{\alpha} \\
\underset{\infty}{\infty}\end{array}$} & Baseline-shortest & 45.50 & 46.95 & 45.40 & 21.86 & 22.61 & 21.83 & 36.80 & 38.01 & 36.74 & 0.69 \\
\hline & Baseline-max-overlap & 49.46 & 41.66 & 44.55 & 23.35 & 19.57 & 20.97 & 39.30 & 33.08 & 35.38 & 0.95 \\
\hline & Original & 49.64 & 41.54 & 44.57 & 23.43 & 19.50 & 20.96 & 39.39 & 32.95 & 35.36 & 0.89 \\
\hline & HERMAN-LOCAL & 48.51 & 42.78 & 44.73 & 22.97 & 20.20 & 21.14 & 38.70 & 34.12 & 35.68 & 0.88 \\
\hline & HERMAN-GLOBAL & 47.88 & 43.52 & 44.79 & 22.66 & 20.56 & 21.17 & 38.26 & 34.79 & 35.80 & 0.92 \\
\hline \multirow{3}{*}{ 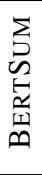 } & Baseline-shortest & 36.78 & 42.26 & 38.71 & 15.61 & $\mathbf{1 7 . 8 7}$ & 16.38 & 29.71 & 33.91 & 31.16 & 0.62 \\
\hline & HERMAN-LOCAL & 38.45 & 40.14 & 38.63 & 16.12 & 16.72 & 16.12 & 30.71 & 31.87 & 30.75 & 0.79 \\
\hline & HERMAN-GLOBAL & 37.99 & 41.59 & 39.06 & 16.24 & 17.70 & 16.65 & 30.59 & 33.28 & 31.36 & 0.81 \\
\hline \multirow{3}{*}{ 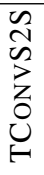 } & Baseline-shortest & 27.43 & 37.28 & 30.99 & 9.84 & 13.49 & 11.15 & 22.43 & 30.41 & 25.32 & 0.45 \\
\hline & Baseline-max-overlap & 30.19 & 34.57 & 31.64 & 10.79 & 12.34 & 11.29 & 24.37 & 27.81 & 25.50 & 0.71 \\
\hline & Original & 30.42 & 34.63 & 31.80 & 10.96 & 12.46 & 11.45 & 24.58 & 27.89 & 25.66 & 0.58 \\
\hline
\end{tabular}

Table 6: Automatic evaluation on the XSum test set. Each of the three horizontal sections reports scores for one of the three abstractive summarization systems: BART, BERTSUM and TCONVS2S. For each system, we present ROUGE scores for the two baseline models, the one original model, and the two variants of our HERMAN model. Baseline-shortest refers to the model that selects the shortest summary. Baseline-max-overlap refers to the model that selects the summary which overlaps the most with the source document in terms of quantity entities . avg-Q denotes the average number of quantity entities per summary.

LOCAL, we also introduce two baseline re-ranking approaches: the first selects the shortest summary from the beam, and the second selects the summary with maximum quantity entity overlap with the source document. The results on the XSum dataset are shown in Table 6. While selecting the shortest summary is a very strong baseline, outperforming all other systems in ROUGE-1/2/L Precision, we can still see that HERMAN-GLOBAL has the best performance in ROUGE-1/2/L Precision and $F_{1}$ despite that baseline. After re-ranking by HERMAN-GLOBAL, $17.27 \%$ originally ranked top summaries produced by BART stayed at the top rank. While BERTSUM had nearly the same, only $9.05 \%$ of the summaries produced by TCONVS2S stayed top-ranked, so if re-ranking leads to improvements, it would be even more helpful in the case of TCONVS2S.

The first thing to note is that the up-ranked summaries have a lower ROUGE Recall than other models. This is common with any model that filters output, since it can exclude items that might otherwise contribute to Recall. ROUGE-1/2/L Precision increases after re-ranking as the verification model ensures summaries with more verified content will be ranked higher in the beam. More verified content also means more tokens appearing in the document and reference summary. Overall, ROUGE-1/2/L $\mathrm{F}_{1}$ score for up-ranked summaries exceeds that of original summaries. To analyze the effect of our systems on quantity entities, we also compute average number of quantity entities per summary for each system. The baseline that selects the summary with maximum quantity entity overlap with the source document, not surprisingly, has very high averages and achieved the highest number for BART. HERMAN-GLOBAL achieves highest average for BERTSUM and TCONVS2S. In BART, it follows the baseline closely at second place. Together with its ROUGE performance, this indicates that our model not only encourages the inclusion of quantity entities in the summary, but also includes them correctly.

To further analyze how our approach affects the distribution of different types of quantity entities, we also computed test set statistics for both original summaries produced by the summarization systems and up-ranked summaries produced by HERMAN-Global. The results are provided in Table 7. Overall, counting all quantity types, we can see that BART encourages the inclusion of quantities the most, for both original and up-ranked summaries, while TCONVS2S has the fewest summaries with quantity entities. However, the number of up-ranked summaries that contain at least one quantity increases the most for TCONVS2S, a 26\% increase compared with the original summaries. This agrees with our prior point that as TCONVS2S has the fewest summaries that remained top after re-ranking, our approach should be most helpful for TCONVS2S. Looking at individual quantity types, the number of summaries containing date or time quantities increases across-the-board through re-ranking. For BERTSUM and TCONVS2S, re- 


\begin{tabular}{|l|rrr|rrr|rrr|}
\hline Quantity & \multicolumn{3}{|c|}{ BART } & \multicolumn{3}{c|}{ BERTSUM } & \multicolumn{3}{c|}{ TCONVS2S } \\
\hline Tyte & Original & Up-ranked & \% diff & Original & Up-ranked & \% diff & Original & Up-ranked & $\%$ diff \\
\hline Time & 3,865 & 4,284 & $11 \%$ & 2,735 & 3,731 & $36 \%$ & 2,347 & 3,747 & $60 \%$ \\
Percent & 208 & 221 & $6 \%$ & 92 & 160 & $74 \%$ & 47 & 75 & $60 \%$ \\
Money & 119 & 118 & $-1 \%$ & 96 & 93 & $-3 \%$ & 93 & 102 & $10 \%$ \\
Quantity & 177 & 166 & $-6 \%$ & 450 & 545 & $21 \%$ & 291 & 379 & $30 \%$ \\
Ordinal & 45 & 37 & $-18 \%$ & 41 & 45 & $10 \%$ & 17 & 16 & $-6 \%$ \\
Cardinal & 1,330 & 1,194 & $-10 \%$ & 959 & 1,057 & $10 \%$ & 1,200 & 1,208 & $1 \%$ \\
\hline All Types & 6,924 & 2,940 & $1 \%$ & 2,327 & 2,580 & $11 \%$ & 2,048 & 2,418 & $18 \%$ \\
\hline
\end{tabular}

Table 7: The statistics of different types of quantity entities on test set summaries for all three abstractive summarization systems: BART, BERTSUM and TCONVS2S. For each system, we provide the number of original summaries and up-ranked summaries that contain at least one instance of the given type of quantity entity. Upranked summaries are produced by HERMAN-GLOBAL. \% diff denotes the percentage difference between the number of up-ranked summaries and the number of original summaries for a given quantity type.

ranking generally increases the number of summaries that contain a specific quantity type, with the exception of percent in BERTSUM and quantity in TCONVS2S where they decreased slightly. We suspect the reason to be that these types are underrepresented in the dataset: Thus, there is insufficient data for the model to learn from. On the other hand, re-ranking in BART leads to more decreases of the number of summaries that contain a specific quantity type. The reason could be that BART already has the highest number of summaries that contain a specific quantity type before re-ranking, and quantity types with a decrease after re-ranking are generally underrepresented types like percent and quantity. Representative types like date and cardinal are still increased through re-ranking.

Human Evaluation Falke et al. (2019) have argued convincingly that ROUGE is inadequate as a measure of hallucination and factual correctness. As such, we have begun to carry out human evaluation. We noted in Section 4 that the XSum reference summary may not be an accurate representation of the source article, in that less than $16 \%$ of the test set reference summaries have quantity tokens that also appear in their corresponding articles. As a result, our human evaluation presented subjects with a text consisting of both the reference summary and the source article, to give subjects a full sense of its contents.

Subjects assessed 40 trials, each consisting of a text followed by two candidate summaries- the original summary produced by the summarization model and the up-ranked summary selected by HERMAN-GlOBAL. These two summaries also satisfied the condition of being very similar except for one quantity entity. The trials comprised 37 randomly selected text-summary pairs that satisfied the additional condition, plus three simple catch trials in which one of the candidate summaries has obvious hallucinated quantities that are never present in the source article, to check whether subjects were paying attention and following the instructions. The order of the trials was randomized for each subject.

In presenting each trial, quantities in the summaries and those with the same type in the text were highlighted to make them easy to find. Subjects were asked to choose the one summary whose highlighted quantity entity is more faithful to the source article. Subjects were also told not to select a summary based on any other factors such as its fluency (i.e., Does the summary sound like wellformed English?). After subjects make a choice of summary, they are also asked whether they think both candidate summaries were equally faithful or equally unfaithful. We will show shortly how subjects can prefer one summary over the other, even while considering both to be faithful (or both to be unfaithful) to the original text. This preliminary experiment was carried out on the Qualtrics platform, with three volunteer subjects. Each subject took between 35 and 45 minutes to finish.

While our results are still preliminary, they provide some evidence that subjects consider the upranked summaries to be more faithful. Specifically, of the 19 trials (other than the three catch trials) where all three subjects agreed on which summary was more faithful, in 12 trials, it was the re-ranked summary (as in Table 8, Article 49), while in only 7 was it the original summary (as in Table 8, Article 4). In all of these cases, the authors agreed with the subjects. Note that no information can be gleaned from those trials in which two of three subjects agreed, since in half of them (9), they agreed on 


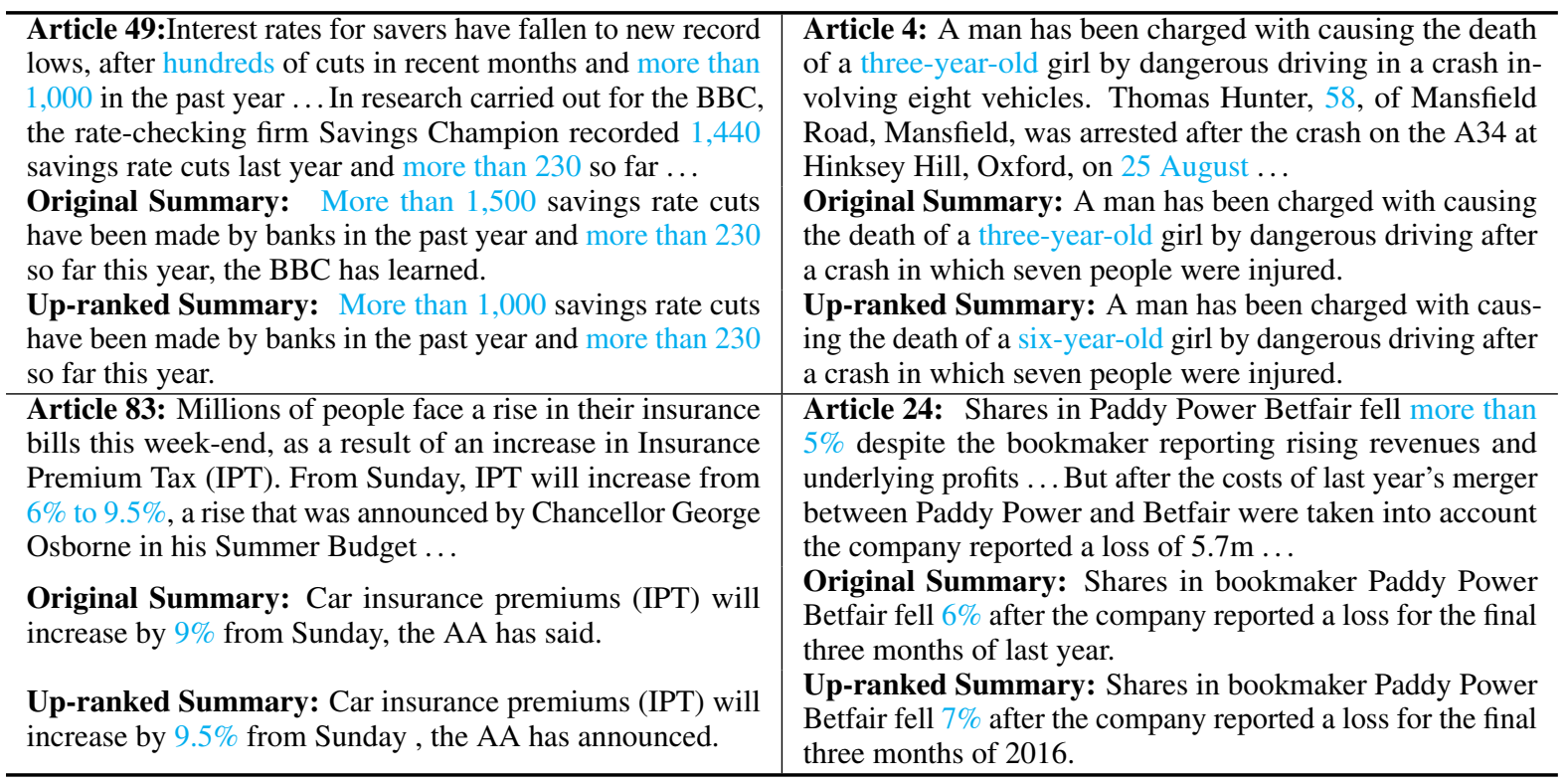

Table 8: Example trials selected from our human evaluation. Quantity entities have been highlighted the same way we did for human evaluation. With article 49 and 83 (containing cardinal and percentage quantities), all subjects agree that the up-ranked summary is more faithful, while with article 4 and 24 (containing date and percentage quantities), all agree that the original summary is more faithful.

the re-ranked summary, and in the other half, they agreed on the original (9).

Finally, the reader may recall that we asked subjects after they selected a summary, whether they considered one summary to be more faithful than the other, or whether both summaries were equally faithful (or equally unfaithful). In 21 trials, at least two subjects indicated that both summaries were equally unfaithful, even if they indicated that they felt one summary was more faithful than the other. Often, it was because its quantity entities were closer to those in the text. For example, Table 8, Article 24 shows that subjects felt the original summary was more faithful since its quantity term $(6 \%)$ was closer to the $5 \%$ that was in the original text, while Table 8 , Article 83 shows them to feel that "by $9.5 \%$ " is closer to the original text than "by $9 \%$ ", even though the quantity in the original text is "to 9.5\%". In over half these trials (13/21), at least two subjects felt that the up-ranked summaries were more faithful.

\section{Conclusions}

In this paper, we addressed the problem of hallucinated quantities in summaries generated by abstractive summarization systems. We introduced HERMAN, a novel approach to recognize and verify quantities in these summaries. Experimental results demonstrate that up-ranked summaries have a higher ROUGE Precision and $F_{1}$ than original summaries produced by a summarization system, indicating our approach reduces hallucinated quantities while still encourage the inclusion of quantity entities. Through human evaluation, we showed that summaries up-ranked by our proposed model are felt to be more faithful than the summaries directly generated by a summarization system.

We also discovered that simple re-ranking strategies, such as the selection of the shortest summary from the beam search, can yield strong performance, if one doesn't care whether a summary communicates specific quantities. We also found that our approach was limited by its use of the XSum dataset, where factual information in the summary sometimes cannot be verified using the article due to the fact that the summary is simply the first sentence of the original article. In the future, we would like to explore the option of incorporating the verification model into training and inference to improve factual correctness of generated summaries.

\section{Acknowledgments}

We would like to thank the anonymous reviewers, Ronald A. Cardenas, and Shashi Narayan for their helpful feedback. We also would like to thank Ronald A. Cardenas, Arlene Casey, Christian Hardmeier, and Javad Hosseini for participating in our human evaluation. 


\section{References}

Dzmitry Bahdanau, Kyunghyun Cho, and Yoshua Bengio. 2015a. Neural machine translation by jointly learning to align and translate. In 3rd International Conference on Learning Representations, ICLR 2015, San Diego, CA, USA, May 7-9, 2015, Conference Track Proceedings.

Dzmitry Bahdanau, Kyunghyun Cho, and Yoshua Bengio. 2015b. Neural machine translation by jointly learning to align and translate. In 3rd International Conference on Learning Representations, ICLR 2015, San Diego, CA, USA, May 7-9, 2015, Conference Track Proceedings.

Michele Banko, Michael J. Cafarella, Stephen Soderland, Matthew Broadhead, and Oren Etzioni. 2007. Open information extraction from the web. In IJCAI 2007, Proceedings of the 20th International Joint Conference on Artificial Intelligence, Hyderabad, India, January 6-12, 2007, pages 2670-2676.

Samuel R. Bowman, Gabor Angeli, Christopher Potts, and Christopher D. Manning. 2015. A large annotated corpus for learning natural language inference. In Proceedings of the 2015 Conference on Empirical Methods in Natural Language Processing, pages 632-642, Lisbon, Portugal. Association for Computational Linguistics.

Ziqiang Cao, Furu Wei, Wenjie Li, and Sujian Li. 2018. Faithful to the original: Fact aware neural abstractive summarization. In Proceedings of the ThirtySecond AAAI Conference on Artificial Intelligence, (AAAI-18), the 30th innovative Applications of Artificial Intelligence (IAAI-18), and the 8th AAAI Symposium on Educational Advances in Artificial Intelligence (EAAI-18), New Orleans, Louisiana, USA, February 2-7, 2018, pages 4784-4791. AAAI Press.

James Clarke and Mirella Lapata. 2008. Global inference for sentence compression an integer linear programming approach. J. Artif. Int. Res. 31(1):399429.

Jacob Devlin, Ming-Wei Chang, Kenton Lee, and Kristina Toutanova. 2019. BERT: Pre-training of deep bidirectional transformers for language understanding. In Proceedings of the 2019 Conference of the North American Chapter of the Association for Computational Linguistics: Human Language Technologies, Volume 1 (Long and Short Papers), pages 4171-4186, Minneapolis, Minnesota. Association for Computational Linguistics.

Tobias Falke, Leonardo F. R. Ribeiro, Prasetya Ajie Utama, Ido Dagan, and Iryna Gurevych. 2019. Ranking generated summaries by correctness: An interesting but challenging application for natural language inference. In Proceedings of the 57th Annual Meeting of the Association for Computational Linguistics, pages 2214-2220, Florence, Italy. Association for Computational Linguistics.
Matt Gardner, Joel Grus, Mark Neumann, Oyvind Tafjord, Pradeep Dasigi, Nelson F. Liu, Matthew E. Peters, Michael Schmitz, and Luke Zettlemoyer. 2018. Allennlp: A deep semantic natural language processing platform. CoRR, abs/1803.07640.

Ben Goodrich, Vinay Rao, Peter J. Liu, and Mohammad Saleh. 2019. Assessing the factual accuracy of generated text. In Proceedings of the 25th ACM SIGKDD International Conference on Knowledge Discovery \& Data Mining, KDD 19, page 166175, New York, NY, USA. Association for Computing Machinery.

Max Grusky, Mor Naaman, and Yoav Artzi. 2018. Newsroom: A dataset of 1.3 million summaries with diverse extractive strategies. In Proceedings of the 2018 Conference of the North American Chapter of the Association for Computational Linguistics: Human Language Technologies, NAACL-HLT 2018, New Orleans, Louisiana, USA, June 1-6, 2018, Volume 1 (Long Papers), pages 708-719. Association for Computational Linguistics.

Karl Moritz Hermann, Tomás Kociský, Edward Grefenstette, Lasse Espeholt, Will Kay, Mustafa Suleyman, and Phil Blunsom. 2015. Teaching machines to read and comprehend. In Advances in Neural Information Processing Systems 28: Annual Conference on Neural Information Processing Systems 2015, December 7-12, 2015, Montreal, Quebec, Canada, pages 1693-1701.

Sepp Hochreiter and Jürgen Schmidhuber. 1997. Long short-term memory. Neural Comput., 9(8): 17351780 .

Matthew Honnibal and Ines Montani. 2017. spaCy 2: Natural language understanding with Bloom embeddings, convolutional neural networks and incremental parsing. http://spacy.io.

Zhiheng Huang, Wei Xu, and Kai Yu. 2015. Bidirectional lstm-crf models for sequence tagging. ArXiv, abs/1508.01991.

Diederik P. Kingma and Jimmy Ba. 2015. Adam: A method for stochastic optimization. In $3 r d$ International Conference on Learning Representations, ICLR 2015, San Diego, CA, USA, May 7-9, 2015, Conference Track Proceedings.

Wojciech Kryscinski, Nitish Shirish Keskar, Bryan McCann, Caiming Xiong, and Richard Socher. 2019a. Neural text summarization: A critical evaluation. In Proceedings of the 2019 Conference on Empirical Methods in Natural Language Processing and the 9th International Joint Conference on Natural Language Processing (EMNLP-IJCNLP), pages 540551, Hong Kong, China. Association for Computational Linguistics.

Wojciech Kryscinski, Bryan McCann, Caiming Xiong, and Richard Socher. 2019b. Evaluating the factual consistency of abstractive text summarization. CoRR, abs/1910.12840. 
John D. Lafferty, Andrew McCallum, and Fernando C. N. Pereira. 2001. Conditional random fields: Probabilistic models for segmenting and labeling sequence data. In Proceedings of the Eighteenth International Conference on Machine Learning (ICML 2001), Williams College, Williamstown, MA, USA, June 28 - July 1, 2001, pages 282-289. Morgan Kaufmann.

Mike Lewis, Yinhan Liu, Naman Goyal, Marjan Ghazvininejad, Abdelrahman Mohamed, Omer Levy, Veselin Stoyanov, and Luke Zettlemoyer. 2019. BART: denoising sequence-to-sequence pretraining for natural language generation, translation, and comprehension. CoRR, abs/1910.13461.

Chin-Yew Lin. 2004. ROUGE: A package for automatic evaluation of summaries. In Text Summarization Branches Out, pages 74-81, Barcelona, Spain. Association for Computational Linguistics.

Hui Lin and Jeff Bilmes. 2011. A class of submodular functions for document summarization. In Proceedings of the 49th Annual Meeting of the Association for Computational Linguistics: Human Language Technologies, pages 510-520, Portland, Oregon, USA. Association for Computational Linguistics.

Yang Liu and Mirella Lapata. 2019. Text summarization with pretrained encoders. In Proceedings of the 2019 Conference on Empirical Methods in Natural Language Processing and the 9th International Joint Conference on Natural Language Processing (EMNLP-IJCNLP), pages 3730-3740, Hong Kong, China. Association for Computational Linguistics.

Annie Louis and Ani Nenkova. 2011. Text specificity and impact on quality of news summaries. In Proceedings of the Workshop on Monolingual Text-ToText Generation, pages 34-42, Portland, Oregon. Association for Computational Linguistics.

Joshua Maynez, Shashi Narayan, Bernd Bohnet, and Ryan T. McDonald. 2020. On faithfulness and factuality in abstractive summarization. CoRR, abs/2005.00661.

Ramesh Nallapati, Feifei Zhai, and Bowen Zhou. 2017. Summarunner: A recurrent neural network based sequence model for extractive summarization of documents. In Proceedings of the Thirty-First AAAI Conference on Artificial Intelligence, February 4-9, 2017, San Francisco, California, USA, pages 30753081. AAAI Press.

Shashi Narayan, Shay B. Cohen, and Mirella Lapata. 2018a. Don't give me the details, just the summary! topic-aware convolutional neural networks for extreme summarization. In Proceedings of the 2018 Conference on Empirical Methods in Natural Language Processing, pages 1797-1807, Brussels, Belgium. Association for Computational Linguistics.
Shashi Narayan, Shay B. Cohen, and Mirella Lapata. 2018b. Ranking sentences for extractive summarization with reinforcement learning. In Proceedings of the 2018 Conference of the North American Chapter of the Association for Computational Linguistics: Human Language Technologies, Volume 1 (Long Papers), pages 1747-1759, New Orleans, Louisiana. Association for Computational Linguistics.

Adam Paszke, Sam Gross, Francisco Massa, Adam Lerer, James Bradbury, Gregory Chanan, Trevor Killeen, Zeming Lin, Natalia Gimelshein, Luca Antiga, Alban Desmaison, Andreas Kopf, Edward Yang, Zachary DeVito, Martin Raison, Alykhan Tejani, Sasank Chilamkurthy, Benoit Steiner, Lu Fang, Junjie Bai, and Soumith Chintala. 2019. Pytorch: An imperative style, high-performance deep learning library. In H. Wallach, H. Larochelle, A. Beygelzimer, F. d Alché-Buc, E. Fox, and R. Garnett, editors, Advances in Neural Information Processing Systems 32, pages 8024-8035. Curran Associates, Inc.

Jeffrey Pennington, Richard Socher, and Christopher D. Manning. 2014. Glove: Global vectors for word representation. In Empirical Methods in Natural Language Processing (EMNLP), pages 1532-1543.

Lance A Ramshaw and Mitchell P Marcus. 1999. Text chunking using transformation-based learning. In Natural language processing using very large corpora, pages 157-176. Springer.

Alexander M. Rush, Sumit Chopra, and Jason Weston. 2015. A neural attention model for abstractive sentence summarization. In Proceedings of the 2015 Conference on Empirical Methods in Natural Language Processing, pages 379-389, Lisbon, Portugal. Association for Computational Linguistics.

Evan Sandhaus. 2008. The new york times annotated corpus. Linguistic Data Consortium, Philadelphia, 6(12):e26752.

Abigail See, Peter J. Liu, and Christopher D. Manning. 2017. Get to the point: Summarization with pointergenerator networks. In Proceedings of the 55th Annual Meeting of the Association for Computational Linguistics (Volume 1: Long Papers), pages 10731083, Vancouver, Canada. Association for Computational Linguistics.

Ilya Sutskever, Oriol Vinyals, and Quoc V. Le. 2014. Sequence to sequence learning with neural networks. In Advances in Neural Information Processing Systems 27: Annual Conference on Neural Information Processing Systems 2014, December 8-13 2014, Montreal, Quebec, Canada, pages 3104-3112.

Ashish Vaswani, Noam Shazeer, Niki Parmar, Jakob Uszkoreit, Llion Jones, Aidan N Gomez, Ł ukasz Kaiser, and Illia Polosukhin. 2017. Attention is all you need. In I. Guyon, U. V. Luxburg, S. Bengio, 
H. Wallach, R. Fergus, S. Vishwanathan, and R. Garnett, editors, Advances in Neural Information Processing Systems 30, pages 5998-6008. Curran Associates, Inc.

Oriol Vinyals, Meire Fortunato, and Navdeep Jaitly. 2015. Pointer networks. In Advances in Neural Information Processing Systems 28: Annual Conference on Neural Information Processing Systems 2015, December 7-12, 2015, Montreal, Quebec, Canada, pages 2692-2700.

Kai Wang, Xiaojun Quan, and Rui Wang. 2019. BiSET: Bi-directional selective encoding with template for abstractive summarization. In Proceedings of the 57th Annual Meeting of the Association for Computational Linguistics, pages 2153-2162, Florence, Italy. Association for Computational Linguistics.

Thomas Wolf, Lysandre Debut, Victor Sanh, Julien Chaumond, Clement Delangue, Anthony Moi, Pierric Cistac, Tim Rault, Rémi Louf, Morgan Funtowicz, and Jamie Brew. 2019. Huggingface's transformers: State-of-the-art natural language processing. CoRR, abs/1910.03771.

Yuhao Zhang, Derek Merck, Emily Bao Tsai, Christopher D. Manning, and Curtis P. Langlotz. 2019. Optimizing the factual correctness of a summary: A study of summarizing radiology reports. CoRR, abs/1911.02541. 


\section{A Supplementary Material}

This appendix provides details of training for our HERMAN model, in addition to experiment settings mention in Section 5. Our HERMAN model has $19,424,661$ parameters in total. On a single GeForce GTX $1080 \mathrm{Ti}$ GPU, with batch size of 32 and using GloVe vectors (6B tokens, uncased) for word embeddings initialization, our HERMAN model need approximately 1 hour to train one epoch. With the same GPU and batch size, HERMAN model with pre-trained base-uncased BERT for word embedding initialization requires 3 hours to train one epoch. We use the Huggingface Transformers library (Wolf et al., 2019) for BERT word embedding initialization.

As mentioned in Section 5, we are using three summarization systems, TCONVS2S, BERTSUM, and BART for getting the beam of summaries to be re-ranked by HERMAN. For BERTSUM, we use the abstractive model variant BERTSUMEXTABS which gets the best performance for XSum. We use the same beam size as reported by the authors. For TCONVS2S, BERTSUM, and BART, beam size used are 10,5 , and 6 , respectively. We did hyperparameter search for $\alpha$ which indicates weight balance between $\mathcal{L}_{Y}$ and $\mathcal{L}_{z}$. Our search space is $[0,1]$, with three configurations, $\alpha=0.33$, $\alpha=0.5$, and $\alpha=0.66$. We choose the best configuration, $\alpha=0.66$, based on the loss on the validation set. 